\title{
Utility of Liver Fatty Acid Binding Protein as a Potential Marker of Tubular Dysfunction in Children with Nephrolithiasis
}

\author{
Katarzyna Taranta-Janusz*, Elżbieta Orłowska and Joanna Poźniak \\ Department of Pediatrics and Nephrology, Medical University of Białystok, Poland
}

*Corresponding author: Katarzyna Taranta-Janusz, Department of Pediatrics and Nephrology, Medical University of Białystok, Poland

\section{ARTICLE INFO}

Received: 慧 April 18, 2019

Published: 幽 April 26, 2019

Citation: Katarzyna T-J, Elżbieta 0, Joanna P. Utility of Liver Fatty Acid Binding Protein as a Potential Marker of Tubular Dysfunction in Children with Nephrolithiasis. Biomed J Sci \& Tech Res 17(3)-2019. BJSTR. MS.ID.003013.

Abbreviations: AKI: Acute Kidney Injury; CKD: Chronic Kidney Disease; eGFR: Estimated Glomerular Filtration Rate; HCa: Hypercalciuria; IL-18: Interleukin-18; L-FABP: Liver Fatty Acid Binding Protein; ROC: Receiver Operating Characteristic; sCre: Serum Creatinine

\section{ABSTRACT}

Objective: Of this study was to evaluate the excretion of the tubular protein liver fatty acid binding protein (L-FABP) as a potential novel biomarker of renal dysfunction in children with nephrolithiasis.

Patients and Methods: We enrolled sixty patients, who were divided into two groups: 1) stone formers; 2) hypercalciuric children. The control study cohort consisted of twenty-eight children with no signs or symptoms of urogenital disease were also enrolled for this study. Urine L-FABP levels were measured using the commercial ELISA kit. Hypercalciuria was defined as a daily urinary excretion of more than $4 \mathrm{mg}$ calcium $/ \mathrm{kg}$ body weight.

Results: The levels of urine L-FABP were higher in the group of children with nephrolithiasis than in controls $(\mathrm{p}<0.01)$. A significant difference was also found between patient groups (stone formers and hypercalciuric) and healthy children with respect to urine L-FABP $(p<0.05)$. Urine L-FABP levels were negatively correlated with estimated glomerular filtration rate evaluated by updated Schwartz formula $(\mathrm{p}<0.01)$.

Conclusion: Increased levels of L-FABP were found in children with nephrolithiasis in comparison to controls $(\mathrm{p}<0.01)$. Although urine L-FABP may be a promising biomarker for early tubular damage, its value in nephrolithiasis warrants further investigation.

Keywords:Children; Liver Fatty Acid Binding Protein; Nephrolithiasis; Renal Function

\section{Introduction}

Nephrolithiasis is one of the most frequent urologic diseases. Recent decades the prevalence of kidney stones in childhood has been on the rise [1]. The reason for this increase is not fully understood, but it has been associated with diet, genetic inheritance, and other environmental factors [2]. However, the limited data available on pediatric nephrolithiasis suggests that there are indeed differences between the formation of stones in children and in adults, which explains the need of further studies to improve prevention strategies.

Urinary stone disease is not just a symptomatic stone, there is a chronic metabolic condition. Experts thought that the symptomatic kidney stone formers seem to be at increased risk for chronic kidney disease. Although kidney stones formers are not commonly identified as the primary cause of chronic kidney disease, they still may be important contributing factors. Early diagnosed impaired renal function enables prior intervention. For these reasons, novel biomarkers for early diagnosis and prognosis of renal impairment are sought. One of these biomarkers, L-Type Fatty Acid-Binding Protein (L-FABP) concentration, may enable early detection of renal function loss.

Several studies have shown the efficacy of urinary biomarkers including Interleukin-18 (IL-18); and liver-Type Fatty Acid Binding Protein (L-FABP) to detect kidney injury before change in serum creatinine [3-5]. New renal biomarkers have the potential to improve both diagnosis and prognosis of patients with impaired renal function.

Fatty Acid-Binding Proteins (FABPs) bind different fatty acids and take on the transport to cellular compartments. Thus, FABPs are involved in lipid metabolism and regulation of gene expression [6]. Several types of FABP, such as Liver Type (L-FABP, FABP1), 
Heart Type (H-FABP,FABP3), and Brain Type (B-FABP, FABP7), have been identified and their functions are tissue-specific [7]. Modified FABP expression patterns were described for prostate, bladder and for renal cell carcinoma, suggesting that FABPs could be used as tumour markers [8]. Until now, we have not had enough information on the role of L-FABP as a marker of the kidney damage in pediatric patients with nephrolithiasis. This study therefore analyzed the urine concentration of L-FABP and whether its excretion adds information to the well-established renal function parameters in pediatric nephrolithiasis.

\section{Patients and Methods}

For the aim of this study we considered sixty pediatric patients with nephrolithiasis who were diagnosed between January 2015 to December 2018 in the Department of Paediatrics and Nephrology, Medical University of Bialystok, Poland. The study was approved by the Ethics Committee of the Medical University of Bialystok, Poland. Children were categorized into two study groups. The first group included 37 children ( 21 boys, 16 girls) with renal stones, aged median 16.1 years (3.83 - 17.9yrs). The criterion adopted for inclusion in the study group 1 was the presence of renal stones on ultrasonographic scan used as the primary procedure. The study group 2 consisted of 23 cases (18 boys, 5 girls; median: 11.8yrs) with hypercalciuria. The control group contained 28 patients (13 boys, 15 girls; median age $12.24 y r s$ ) who were healthy without any signs or symptoms of urogenital disease.

Interpreted clinical features were age, gender, and renal function (serum creatinine, glomerular filtration rate estimated by the Schwartz formula - eGFR). All patients underwent a thorough history and physical examination, and laboratory evaluation: twenty-four urine sample was analysed for levels of calcium.
Hypercalciuria was defined as a daily urinary excretion of more than $4 \mathrm{mg}$ calcium $/ \mathrm{kg}$ body weight. After at least 12 hours fasting venous blood was analysed for creatinine; morning spot urine was collected for urine analysis. Exclusion criteria: associated anomalies including vesicoureteral reflux, previous operation in the urinary system and other deformations of the external genital organs, deformations in the lower part of the ureter, bladder and urethra.

The urine was collected between 7 and 8 am from morning sample. Urine measurements of L-FABP were performed in freshly voided midstream samples frozen at the temperature $-80^{\circ} \mathrm{C}$. Patients with urinary tract infection were excluded from the examination. L-FABP was measured in the urine using ELISA commercially available kit (BioVendor Inc.,Brno, Czech Republic), according to manufacturer instructions. Urine L-FABP levels were expressed in $\mathrm{ng} / \mathrm{mL}$. All data were analyzed using SPSS Statistics 12.0 (StatSoft, Tulsa, OK, USA). A Receiver Operating Characteristic (ROC) curve was used to determine the cut-off values of estimated markers that gave the best sensitivity and specificity.

\section{Results}

Demographic data were established and found to be similar between the two groups. Table 1 shows the characteristics and laboratory outcomes of all 88 patients from the study and the control groups. Age, body weight, height, serum creatinine, and eGFR did not significantly differ among the groups. At the time of diagnosis, obstructive nephropathy was present in a total of $18.3 \%$ of study patients. Levels of urine L-FABP were significantly increased when compared to the control group ( $\mathrm{p}=0.0062$; Table 1 ). Our analysis revealed no significant differences in urine $\beta 2$-microglobulin levels between the studied and control subjects ( $p>0.05)$.

Table 1: Anthropometric and clinical characteristics of the patients and reference group.

\begin{tabular}{|c|c|c|c|}
\hline & Study group $\mathbf{n = 6 0}$ & Control group n= 28 & p value \\
\hline Age & $14.05(9.02-16.68)$ & $12.24(9.92-15.74)$ & 0.705 \\
\hline Body weight $(\mathrm{kg})$ & $49.5(27.25-59.0)$ & $45.05(28.4-57.2)$ & 0.672 \\
\hline Height $(\mathrm{cm})$ & $160.5(32.5-169.5)$ & $153.7(135.5-165.0)$ & 0.602 \\
\hline sCre $(\mathrm{mg} / \mathrm{dL})$ & $0.59(0.34-0.75)$ & $0.54(0.44-0.75)$ & 0.687 \\
\hline eGFR $\left(\mathrm{mL} / \mathrm{min} / 1.73 \mathrm{~m}^{2}\right)$ & $135.33(112.44-165.73)$ & $120.52(100.96-143.92)$ & 0.081 \\
\hline Urine $\beta 2$-microglobulin $(\mu \mathrm{g} / \mathrm{mL})$ & $0.08(0.05-0.12)$ & $0.008(0.007-0.022)$ & 0.563 \\
\hline Urine L-FABP $(\mathrm{ng} / \mathrm{mL})$ & $12.12(0.02-23.17)$ & $6.31(0-12.2)$ & 0.0062 \\
\hline Urine L-FABP/Cr $(\mathrm{ng} / \mathrm{mg} \mathrm{Cr})$ & $0.01(0.005-0.15)$ & $0.008(0.007-0.02)$ & 0.02 \\
\hline
\end{tabular}

Note: Data are presented as the median with the interquartile range [IQR; Q1 (lower quartile) and Q3 (upper quartile)] in parenthesis, unless otherwise indicated eGFR, estimated glomerular filtration rate; sCre serum creatinine.

Urine L-FABP levels in stone formers were significantly greater than in hypercalciuric and control groups $(\mathrm{p}<0.05)$. Patients with hypercalciuria also presented with increase in L-FABP urine excretion in comparison to healthy participants $(\mathrm{p}<0.05)$ (Figure 1). Further tests were carried out to identify correlations of estimated marker with parameters of renal function. There was no association of estimated marker with serum creatinine or $\beta 2$ microglobulin. Urine L-FABP displayed only significant negative correlation with eGFR ( $r=-0.36$; $p=0.005)$ (Figure 2$)$. ROC analyses were performed in order to define the diagnostic efficiency of renal function parameters (urine L-FABP, sCre, eGFR) in identifying children with obstructive nephropathy among all examined children with nephrolithiasis. In this analysis area under the curve (AUC) for urine L-FABP was 0.642 , for sCre 0.463 and for eGFR 0.525 (Table 2). 
Table 2: Receiver Operating Characteristic (ROC) analyses for urine L-FABP, Serum Creatinine (sCre) and eGFR levels in children with obstructive nephropathy among all examined children.

\begin{tabular}{|c|c|c|c|c|}
\hline & AUC & SE & $\mathbf{- 9 5 \%}$ & $\mathbf{+ 9 5 \%}$ \\
\hline Urine L-FABP $(\mathrm{ng} / \mathrm{mL})$ & 0.642 & 0.094 & 0.457 & 0.828 \\
\hline sCre $(\mathrm{mg} / \mathrm{dL})$ & 0.463 & 0.088 & 0.291 & 0.635 \\
\hline eGFR $(\mathrm{mL} / \mathrm{min} / 1.73 \mathrm{~m} 2)$ & 0.463 & 0.088 & 0.291 & 0.635 \\
\hline
\end{tabular}

Note: AUC- Area Under Curve; SE- Standard Error

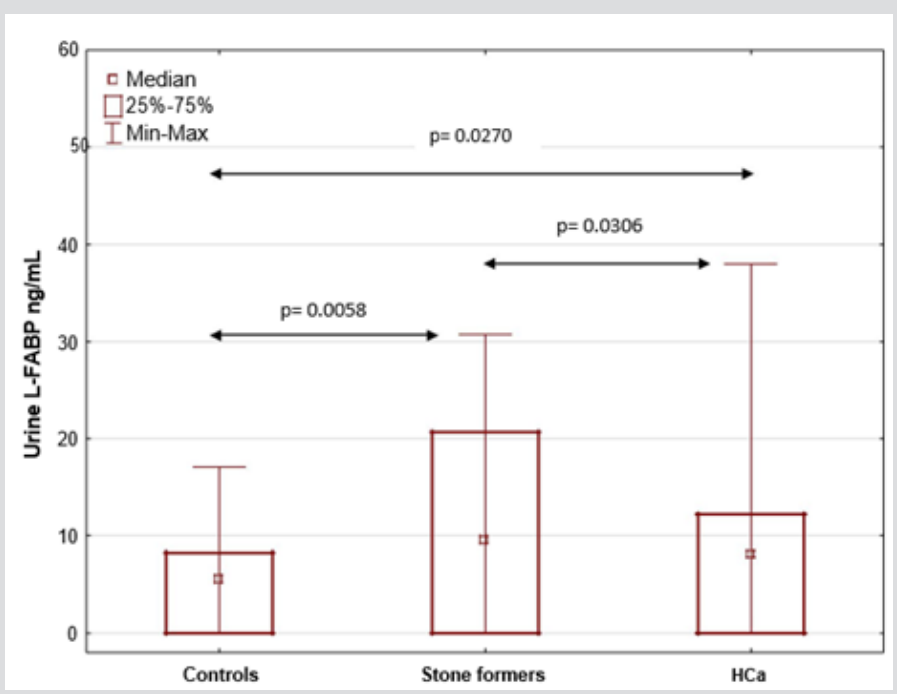

Figure 1: Comparison of urine L-FABP levels in patients with Hypercalciuria (HCa), stone formers and healthy participants.

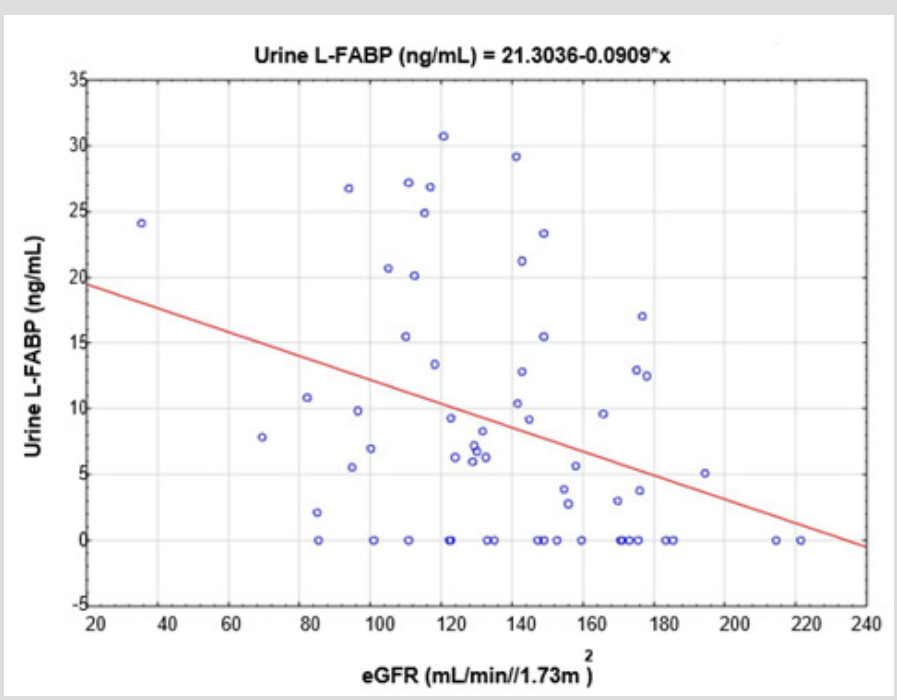

Figure 2: Scatter plot for urine L-FABP $(\mathrm{ng} / \mathrm{mL})$ versus eGFR levels in all studied patients.

\section{Discussion}

The present study was designed to explore urine L-FABP levels and their relation to the kidney function in children with nephrolithiasis in comparison to healthy children. Firstly, urine L-FABP concentration has been found to be significantly higher in nephrolithiasis patients when compared to the healthy participants. In addition, urine L-FABP excretion was significantly higher in patients when divided into groups with stones and hypercalciuria in connection to their healthy counterparts. Moreover, our results showed that urine L-FABP values in study group correlated negatively to the parameter of kidney function, such as eGFR.

These results are different from findings by Dede and colleagues [9], who evaluated the damage effects of retrograde intra-renal surgery on kidney tissue in adult patients with renal calculi less than $2 \mathrm{~cm}$. They found that mean levels of L-FABP did not change significantly on the pre-operative day, the post-operative second hour, and post-operative first day. Similarly, Daggülli and co-authors [10] showed, in 29 adult patients who underwent percutaneous 
nephrolithotomy, no statistically significant difference in urine L-FABP values secondary to surgical intervention.

Clinical and experimental lines of evidence leave no doubt about an independent association of nephrolithiasis with Acute Kidney Injury (AKI) and Chronic Kidney Disease (CKD). Therefore, urine measurements of renal damage newer biomarkers seemed to be useful in the diagnosis of nephrolithiasis-associated AKI, and as predictors of underlying CKD associated risk factors. There are also some experimental animal studies designed to examine L-FABP role of AKI and sepsis [11]. While changes in L-FABP as a biomarker for kidney injury have been described in children with steroidsensitive nephrotic syndrome [12], with vesicoureteral reflux [13], there still are no studies involving the pediatric population with nephrolithiasis.

Furthermore, contradictory to our study, in report of Nishida and colleagues [12] in pediatric nephrotic syndrome L-FABP significantly correlated with urinary $\beta 2$-microglobulin. In summary, this study showed that children with nephrolithiasis were characterised by increased L-FABP. We believe that our study showed that urine L-FABP concentration may be a useful marker in the evaluation of early renal dysfunction due to tubular damage caused by nephrolithiasis. This declaration is supported by data from ROC analyses, which revealed quite good profile in identifying children with obstructive nephropathy among all examined children. There are limitations to this study that should be mentioned. This a single center study with relatively small number of patients, which does not allow us to draw an unequivocal conclusion.

\section{Conclusion}

It is believed that this is the first study to evaluate urine L-FABP levels in children with nephrolithiasis which might help the management and prognosis of tubular dysfunction. Further studies are needed for assessment if L-FABP may be used as a marker for early screening of patients with nephrolithiasis and for determining renal dysfunction and poor prognosis.

\section{Acknowledgement}

The authors declare no conflicts of interest regarding the content herein. The research was supported by a grant (N/ST/ ZB/17/006/1141) from the Medical University of Bialystok, Poland.

\section{References}

1. Asplin JR (2008) Nephrolithiasis: Introduction. Semin Nephrol 28: 9798.

2. Amancio L, Fedrizzi M, Bresolin NL, Penido MG (2016) Pediatric urolithiasis: Experience at a tertiary care pediatric hospital. J Bras Nefrol 38(1): 90-98.

3. Lin X, Yuan J, Zhao Y, Zha Y (2015) Urine interleukin-18 in prediction of acute kidney injury: A systemic review and meta-analysis. J Nephrol 28: 7-6.

4. Ho J, Tangri N, Komenda P, Kaushal A, Sood M, et al. (2015) Urinary, plasma, and serum biomarkers' utility for predicting acute kidney injury associated with cardiac surgery in adults: a meta-analysis. Am J Kidney Dis Off J Natl Kidney Found 66: 993-1005.

5. Parr SK, Clark AJ, Bian A, Shintani AK, Wickersham NE, et al. (2015) Urinary L-FABP predicts poor outcomes in critically ill patients with early acute kidney injury. Kidney Int 87: 640-648.

6. Das R, Hammamieh R, Neill R, Melhem M, Jett M (2001) Expression pattern of fatty acid-binding proteins in human normal and cancer prostate cells and tissues. Clin Cancer Res 7: 1706-1715.

7. Storch J, Thumser AE (2010) Tissue-specific functions in the fatty acidbinding protein family. J Biol Chem 285: 32679-32683.

8. Ohlsson G, Moreira JM, Gromov P, Sauter G, Celis JE (2005) Loss of expression of the adipocyte-type Fatty Acid-Binding Protein (A-FABP) is associated with progression of human urothelial carcinomas. Mol Cell Proteomics 4: 570-581.

9. Dede O, Dağguli $M$, Utanğaç $M$, Yuksel $H$, Bodakcı MN, et al. (2015) Urinary expression of acute kidney injury biomarkers in patients after RIRS: It is a prospective, controlled study. Int J Clin Exp Med 8(5): 81478152.

10. Daggülli M, Utangaç MM, Dede O, Bodakci MN, Hatipoglu NK, et al. (2016) Potential biomarkers for the early detection of acute kidney injury after percutaneous nephrolithotripsy. Ren Fail 38(1): 151-156.

11. Doi K, Noiri E, Sugaya T (2010) Urinary L-type fatty acid-binding protein as a new renal biomarker in critical care. Curr Opin Crit Care 16(6): 545549.

12. Nishida M, Kawakatsu H, Hamaoka K (2018) Urinary liver-type fatty acid-binding protein in pediatric nephrotic syndrome and tubular dysfunction. Pediatr Int 60(5): 442-445.

13. Benzer M, Tekin Neijmann S, Gültekin ND, Uluturk Tekin A (2017) Urinary L-FABP as a marker of vesicoureteral reflux in children: Could it also have a protective effect on the kidney? Int Urol Nephrol 49(1): 1-12.

\section{ISSN: 2574-1241}

DOI: 10.26717/BJSTR.2019.17.003013

Katarzyna Taranta-Janusz. Biomed J Sci \& Tech Res

This work is licensed under Creative

Commons Attribution 4.0 License

Submission Link: https://biomedres.us/submit-manuscript.php

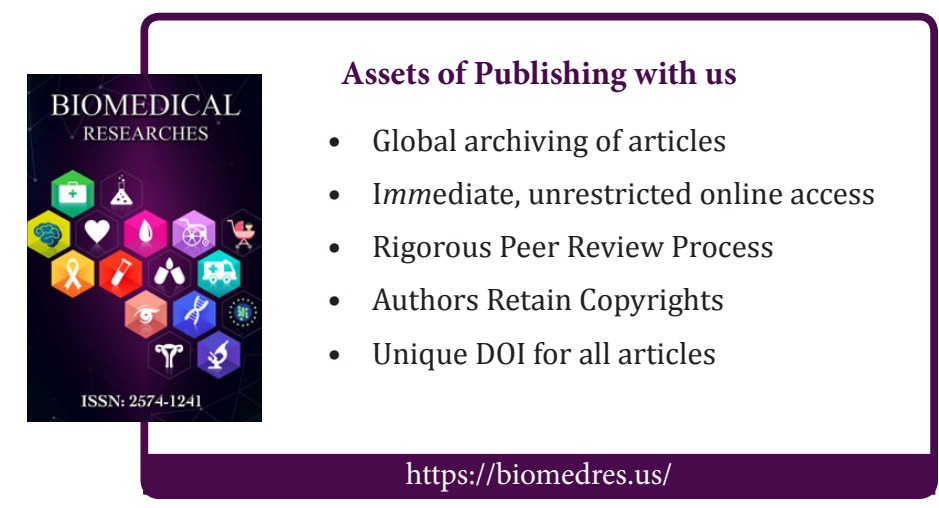

